\title{
Discordances momentanées. Analyse comparative
}

\section{Geneviève Bédoucha}

\section{OpenEdition}

Journals

Édition électronique

URL : https://journals.openedition.org/tc/1428

DOI : $10.4000 /$ tc. 1428

ISSN : 1952-420X

\section{Éditeur}

Éditions de l'EHESS

\section{Édition imprimée}

Date de publication : 1 avril 2003

ISSN : 0248-6016

\section{Référence électronique}

Geneviève Bédoucha, «Discordances momentanées. Analyse comparative », Techniques \& Culture [En ligne], 40 | 2003, mis en ligne le 18 mai 2006, consulté le 29 septembre 2022. URL : http:// journals.openedition.org/tc/1428; DOI : https://doi.org/10.4000/tc.1428

Ce document a été généré automatiquement le 29 septembre 2022

Tous droits réservés 


\title{
Discordances momentanées. Analyse comparative
}

\author{
Geneviève Bédoucha
}

1 Je traiterai ici de deux sociétés pour le moins contrastées. L'une en effet se trouve en zone aride, l'autre en zone humide. Toutes deux ont mis en œuvre des systèmes hydrauliques, systèmes techniques complexes de gestion des eaux -j'insiste sur ce qualificatif et y reviendrai-, mais à l'évidence fort différents puisque, dans le premier cas, celui d'une société oasienne du Sahara, il s'agit d'un système de répartition des eaux d'une source qui seul rend possible une agriculture irriguée, tandis que dans l'autre, celui d'une société, située en Brenne, dans le Berry, au cœur de la France, il s'agit d'une exploitation d'étangs dans un but piscicole; on ne saura toutefois comprendre le fonctionnement de ce dernier système sans aussi mesurer la valeur de son insertion dans le monde rural alentour : les étangs ont une importance essentielle, notamment pour l'élevage, et une complémentarité ancienne existe entre exploitation des étangs, agriculture et élevage, à la base du système social jusqu'à nos jours, malgré ce qu'il est convenu d'appeler aujourd'hui une forte déprise agricole. Avec cet exemple, nous verrons, entre autres, comment la nécessité de gestion d'un système technique structure véritablement une société au niveau d'une région tout entière.

2 Pour les deux sociétés, je ferai apparaître clairement le lien étroit qui existe entre le technique, plus spécifiquement un système technique de gestion des eaux, et le social ${ }^{1}$. Dans les deux cas, si, par le biais de leurs coutumiers, ces sociétés établissent des règles, le système, en ses moments forts, saison d'été pour le système d'irrigation, saisons d'automne et d'hiver pour le système d'étangs, repose largement sur différents arrangements au jour le jour, de saison à saison. Mais je dirai que d'emblée le seul fait qu'il y ait, ici et là, une gestion hydraulique pourrait suffire à rendre l'analyse comparative possible et légitime.

3 Cette assertion plus générale de R. Cresswell (1996) selon laquelle « il ne s'agit pas de prétendre trouver tout le social dans le technique ", mérite d'être nuancée, car il existe bien, selon moi, une hiérarchie dans les techniques : s'il y a toujours forte interaction avec le social, relation essentielle, sujette d'ailleurs à variations, toutes les techniques 
ne me semblent pas avoir le même statut, le même niveau d'importance ${ }^{2}$. Quelle que soit la société concernée, lorsqu'il y a système de gestion des eaux, les techniques en jeu, captage, retenue, dérivation, distribution etc., sont au centre d'une gestion large qui touche le groupe dans son entier. Qu'il s'agisse de systèmes mis en place pour l'irrigation ou pour l'exploitation d'étangs, les eaux sont agencées en un vaste réseau qui se ramifie jusqu'au plus obscur, au plus ténu du terroir, au plus intime du social. Par un dysfonctionnement du système, un large groupe d'hommes, si ce n'est tous, sont concernés. J'avancerais volontiers que, dans ces techniques-là, on peut prétendre discerner une grande part des autres registres du social.

4 J'aurai, dans mon analyse, à démontrer pourquoi, à mes yeux, les sociétés dont il sera question ici sont, plus largement encore que je ne viens de l'énoncer, comparables, et me proposerai d'examiner les discordances en termes d'efficacité apparues ici et là, à un moment ou à un autre de leur histoire, entre système technique et société, les perceptions et les réactions de ces sociétés face à ces discordances, parfois flagrantes pour les hommes eux-mêmes. La société brennouse nous offre la possibilité d'analyser sur le vif ces réactions du groupe qui perçoit la menace, et en pressentant déjà les effets, tente d'y parer. Tout au long de cette analyse transparaîtront les rapports dialectiques qui existent nécessairement entre les techniques et la société dans son ensemble.

5 Pour la première des sociétés que je présenterai, une société oasienne du Sahara tunisien, l'eau est la ressource rare, essentielle, déterminante et la terre n'a de valeur qu'en second lieu, parce que vivifiée par l'eau qui fait alors l'objet d'une appropriation; malgré les claires réticences de l'islam en la matière, les droits d'eau sont incontestablement des droits privés, transmis au sein du groupe et, indépendants de la propriété de la terre, ils peuvent être soumis à toutes sortes de transactions.

6 Ici, et dans bien d'autres sociétés où l'eau est une ressource rare, elle se voit répartie non pas en volume et topographiquement mais dans le temps, et c'est une organisation gentilice qui préside à sa distribution : un groupe lignager a le privilège de la jouissance exclusive de l'eau pendant un temps donné, privilège qui aura été négocié ou imposé aux autres groupes en présence. Il y a ainsi au départ emprise de lignages sur l'eau et dès lors sur des portions de territoire, véritable projection dans l'espace des groupes et de leurs segmentations. Ce qui leur est le plus précieux, chacun d'eux semble vouloir le gouverner dans l'intimité, avant tout s'en débrouiller seul. Cette inscription des groupes dans le terroir, cette concordance parfaite entre ordre technique et ordre social renvoient à la représentation idéale qu'ils ont d'eux-mêmes.

7 Voilà très rapidement pour les principes, éminemment sociaux, qui président à un système technique de répartition des eaux en zone aride; bien entendu, quantité d'autres exemples pourraient l'illustrer.

8 Le système peut atteindre une grande ampleur : dans le groupe d'ensemble des oasis sur lequel j'ai travaillé autrefois, c'est un système cyclique qui régissait la répartition d'une source abondante entre quatre villages; toute l'eau de la source était amenée en alternance vers le terroir de chacun des villages pour un temps déterminé -un temps évidemment soumis à des rapports de force et constamment menacé, mais le principe était celui-là. Que l'eau de la source soit répartie à moitié entre les villages groupés deux à deux, toute la force du débit de la source n'en restait pas moins dirigée exclusivement vers chacun d'entre eux, puis au sein de chacun d'eux, une répartition cyclique, dans le temps toujours, se faisait entre les groupes lignagers. 
9 Dans cette logique, sociale à l'évidence, de la répartition de l'eau, où se repère l'efficacité technique? Elle ne semble pas exister en tant que telle. L'efficacité est d'abord, avant tout, sociale; du moins ne semble-t-elle pas du point de vue des hommes avoir d'autre définition que sociale. Et s'il y a efficacité technique -le système fonctionne incontestablement-, elle est possible parce que sociale: le curage des fossés, l'entretien des ouvrages, le contrôle de la pulsation de l'eau mais aussi les litiges qui peuvent surgir (jusqu'aux conflits les plus larges et leurs répercussions comme le détournement abusif de l'eau par l'un des villages depuis la tête de la source ou même par un seul des lignages, et la pénurie qui en résulte), le groupe, à ses différents niveaux, intervillageois, villageois ou lignager, choisit de les gérer en son sein. Aux différents niveaux, la solidarité du groupe social est la meilleure façon de préserver l'efficacité technique du système, la meilleure garantie que le système fonctionne techniquement.

10 Mais les hommes doivent également se plier aux exigences techniques du système luimême, auxquelles ils ne peuvent se dérober. Un exemple éloquent est le curage de la source partagée. Pour la préservation du bien commun, aux moments les plus conflictuels de leur histoire, les hommes se doivent d'oublier leurs dissensions, parfois même de faire taire les armes; le curage annuel réunit les hommes des quatre villages.

11 Par la rigueur qu'il exige pour un fonctionnement efficace, le système technique rejaillit à son tour sur l'ensemble du social qu'il contribue intimement à forger. Le système vise à la perfection et il a ses garde-fous, exhérédation des femmes, indivision et maîtrise sans faille du groupe sur son terroir. Ordre technique, ordre juridique, ordre social ne font qu'un.

12 Cela ne dure qu'un temps : du fait de conjonctures historiques diverses, de la mobilité des groupes, de leur dynamisme interne, des intérêts privés peuvent se faire jour et s'affirmer, des tensions surgir. La cohésion des lignages s'en trouve amoindrie, le contrôle des droits d'eau par chacun des lignages et leur maintien au sein du groupe deviennent dès lors difficiles, puis un jour, impossibles. Déjà au XIXe siècle, des initiatives individuelles de ventes d'eau permettent de s'interroger quant à l'efficacité d'un contrôle gentilice de l'eau. Progressivement, le groupe perd la maîtrise de l'eau et des terres; les patrimoines lignagers, à l'origine d'un seul tenant, se démantèlent. Un divorce se dessine et s'accentue entre le lignage, son tour d'eau, sa projection dans un espace qui est le sien : le tour lignager en vient même à ne plus correspondre à un quartier spécifique du terroir. La répartition de l'eau devient d'une grande complexité et coûteuse pour les hommes en eau et en efforts. Mais quelle que soit la dispersion des jardins dans le terroir et l'imbrication des jardins des différents groupes, le privilège lignager d'utiliser toute l'eau pendant une durée déterminée demeure.

Ce qui m'a paru intéressant alors, d'autant qu'on a une possibilité déjà de comparaison au sein du même système technique entre les villages, c'est d'observer de quelle façon les hommes réagissent face à ce décalage qu'ils subissent pour un temps entre un système technique mis en place à un moment donné de leur histoire et une société qui ne semble plus toute s'y retrouver, à laquelle le système semble ne plus correspondre, comme s'il lui était extérieur, « face à elle ».

14 Il est instructif de constater que, pour l'ensemble des quatre villages, puis ultérieurement pour deux d'entre eux, les innovations apportées au système technique d'irrigation vont venir de l'extérieur, d'un ordre étranger à la société locale, précisément, l’ordre colonial. 

d'une rationalité économique qui sont tout à fait étrangères à la société locale. Il clair que l'intervention coloniale est elle-même d'autant plus aisée qu'il y a, au sein des deux villages concernés, des tensions fortes et des enjeux de pouvoir, plus précisément des stratégies d'un groupe, arrivé plus tardivement dans la région, plus moderniste et qui, n'ayant pas la légitimité de l'ancienneté, a tout intérêt à l'instauration d'un nouvel ordre technique; groupe sur lequel à l'évidence le pouvoir colonial va pouvoir compter. À l'appui d'enjeux plus subtils et non énoncés, les arguments de la société coloniale, moindre déperdition d'eau par évaporation ou par infiltration ${ }^{4}$, n'étaient pas pris en compte localement... sans doute, face à d'inextricables situations, les groupes auraientils été amenés, à un moment ou à un autre, à agir, mais beaucoup plus tard, comme cela a été le cas dans les deux autres villages.

Justement, ce qui est très révélateur pour notre propos quant à l'analyse des décalages qui peuvent apparaître entre système technique et société globale, c'est qu'en revanche, dans ces deux autres villages qui font partie du même système hydraulique d'ensemble, là où il n'y a pas eu intervention, ni intrusion claire d'un ordre étranger au sien, la société va continuer d'évoluer dans le même sens -le phénomène d'individualisation qui est apparu s'affirmera toujours plus, avec, dès lors, mobilité accrue des droits d'eau et des terres- et le décalage s'accentuer, sans que pour autant les hommes interviennent dans un système technique de gestion de l'eau (le système de rotation lignagère dans le temps) mis en place par leurs ancêtres. Ils sont pourtant confrontés à des situations socialement exténuantes : du fait des multiples transactions de terres, le parcours de l'eau, pour rejoindre les jardins des membres d'un même lignage, est devenu un vrai dédale. Mais les hommes s'en accommodent, ils se débrouillent. Les nombreux arrangements qu'autorise le système et qui permettaient un fonctionnement efficace, permettent maintenant sa survie. Et alors que, tout près de là, dans les deux villages voisins, le changement a eu lieu au tout début du siècle, pour eux, cela va se prolonger durant des décennies: ce n'est que dans les années 1960, lorsqu'ils auront entendu et admis les arguments sur la rationalité économique véhiculés par les discours des différents techniciens et experts modernistes formés à l'école occidentale, qu'ils prendront la décision, eux-mêmes cette fois, de transformer le système technique de répartition de l'eau et d'inaugurer à leur tour une rotation topographique, sans que plus personne au sein des groupes ne trouve d'argument à opposer.

Ainsi, on assiste là et pendant des décennies à une véritable pesanteur, une sorte d'inertie des techniques de distribution de l'eau, un décalage flagrant des techniques avec la société. Au point que j'avais pu parler de « vestiges archéologiques » (Bédoucha 1987), la répartition des eaux jouant parfois comme véritable « matériau » qu'il nous est donné de voir et à partir duquel se décrypte l'ordre social ancien. Le moins que l'on puisse dire, c'est que l'on ne trouve pas à ce moment de l'histoire de ces sociétés 
oasiennes dans une situation d'« interaction forte » -pour reprendre l'expression de Cresswell (1996) - entre techniques et société.

Un exemple, extrême aux yeux mêmes des oasiens, et souvent évoqué par eux, était celui de la rotation d'une autre source que celle dont il s'est agi jusque là, qui gardait si fortement la marque de l'ancienne préséance d'un lignage disparu. Le système voyait trois tours alterner: le tour de Shbîb ouvrait le cycle d'été, puis suivaient les deux autres tours; mais, trace d'un extraordinaire privilège, chaque $12 \mathrm{du}$ mois, le tour de Shbîb venait interrompre le tour en cours, où qu'il en fût. Les autres groupes s'inclinaient et cédaient la place. Pour les oasiens qui ne cessaient de s'y référer avec une véritable fascination, cet exemple restait emblématique de la toute puissance lignagère inscrite dans la répartition même des eaux, l'image d'une suprématie incontestée: à un moment où les lignages semblaient se dissoudre, l'excellence lignagère s'y exprimait encore sur un mode paroxystique qui prenait alors valeur de symbole pour les hommes.

Mais pour en revenir au système technique d'ensemble de la source analysé précédemment, comment comprendre qu'ait pu perdurer une telle inertie? Si ce n'est justement que le système technique de répartition de l'eau par rotation lignagère dans le temps, désormais sorte de coquille vide, conservait cependant, par sa portée symbolique, une incontestable efficacité sociale pour des lignages, eux, toujours bien présents et qui, malgré un développement évident de l'individualisme et de la division des biens, exaltaient toujours aussi vivement la force de l'appartenance lignagère. Une coquille vide, mais non vidée de sens et que l'on préservera, jusqu'aux limites du supportable, tant que cela sera encore aménageable, et sur ce point, les hommes peuvent aller très loin.

21 Ainsi, un long moment, la société a eu affaire à un système technique qui ne correspondait plus à rien, si ce n'est, et la réserve n'est pas moindre, qu'il renvoyait à la représentation idéale qu'elle gardait d'elle-même, à laquelle les hommes ont toujours du mal à renoncer.

22 J'évoquerai maintenant une société très différente, située dans une région bien spécifique, en Brenne, au centre de la France : un espace de quelque 50000 hectares, enserré entre deux rivières, auquel ses habitants donnent des limites précises, et où se trouve une très forte densité d'étangs, autour d'un millier, qui recouvrent une surface d'environ 9000 hectares dont l'eau, profitant d'une faible déclivité naturelle et par tout un réseau de fossés communiquant entre eux, se jette dans l'une ou l'autre des deux rivières. L'ensemble solidaire forme système.

23 L'observation très précise, et l'analyse que j'ai menée du fonctionnement du système technique d'exploitation des étangs et des perturbations récentes qui y sont apparues, montrent que la gestion des eaux dans une région comme la Brenne est également, aussi paradoxal que cela puisse paraître pour une région connue comme zone humide, une gestion de la rareté des eaux ${ }^{5}$.

Cette rareté des eaux l'est à double titre, je ne pourrai ici qu'être très brève :

- pour les propriétaires d'étangs dans le cadre de l'exploitation d'étangs en vue d'une pratique de la pisciculture ${ }^{6}$;

26 - pour la société paysanne alentour qui se révèle par ailleurs étroitement liée à l'exploitation même des étangs et dont on peut dire dès lors qu'elle fait partie du monde des étangs; la saison d'été est très sèche en Brenne: pour les agriculteurs- 
éleveurs, les hommes de la terre, autrefois métayers chez les propriétaires des domaines, aujourd'hui fermiers ou petits propriétaires, l'eau des étangs et les possibilités de pacage des terres alentour sont un appoint essentiel, vital pour leurs exploitations. Et les étangs ont longtemps été la propriété exclusive des propriétaires : les baux à ferme ne les mentionnent jamais comme part des biens affermés.

Cette rareté de l'eau est déterminante. Les fortes tensions qui ne manquent pas de se produire dans son usage ont amené à l'aménagement au cours des siècles d'un système technique de gestion, et à sa régulation par un droit coutumier auquel on continue de se référer.

En cela également, les deux sociétés présentées ici me paraissent comparables : ordres technique, juridique, symbolique s'y révèlent, de façon exemplaire, intimement mêlés.

Pour ces deux sociétés, aussi contrastées que possible, l'eau aura été dans les deux cas, du fait de cette rareté soulignée, on l'aura compris pour la première, et j'ai maintenant à le démontrer pour la seconde, une ligne utile, fructueuse de déchiffrement du social.

Les termes de "chapelet ", de "lignée ", de "chaîne ", désignent ces suites denses d'étangs et la façon dont ils s'agencent dans l'espace. Ces termes, à forte charge symbolique ne sont pas, on le pressent, employés indifféremment; qu'il me suffise ici de signaler que propriétaires ou experts, gardes, agriculteurs ne recourent pas toujours aux mêmes désignations (même si elles sont entendues de tous et si prédomine aujourd'hui le terme de "chaîne ») : les perceptions des rapports sociaux qu'ont les hommes selon leur appartenance se projettent dans l'espace...

31 Pour une bonne organisation du système d'ensemble, les étangs doivent être lentement vidés, jusqu'à ce qu'il ne reste à une date précise, convenue longtemps à l'avance avant la saison avec le négociant dont l'équipe viendra pêcher l'étang à l'aube du jour fixé, qu'un très faible volume d'eau où se trouve concentré le poisson qui peuple l'étang. La " mise en tire " qui désigne ce vidage progressif, ainsi que la "mise en pêche » qui intervient juste avant le jour fixé, la veille précédant la pêche, sont des opérations délicates, soumises à quantité d'aléas. Chacun a sa façon de faire et de "sentir " les choses. Certains notent scrupuleusement l'évolution du niveau des eaux, utilisent des repères précis qu'ils retrouvent chaque année, d'autres surveillent du regard et n'ont recours aux repères que les tout derniers jours, voire les toutes dernières heures... Mais il y a là, dans tous les cas, tout un savoir mis en œuvre : une évaluation et un dosage de l'écoulement des eaux qui supposent une connaissance fine des temps et des débits selon la configuration de l'étang, sa profondeur, le type et le nombre des ouvrages mis en place. Un manque d'expérience, un défaut de vigilance durant la dernière nuit peuvent contraindre à annuler la pêche. Certains étangs exigent que l'on débute la mise en tire plusieurs semaines, jusqu'à un mois et demi pour les très grands étangs, avant le jour de la pêche qu'une pluie, un gel subit la veille, peuvent éventuellement compromettre.

32 Si je souligne cette difficulté de mise en tire, de ce vidage, en douceur, de l'étang, c'est pour suggérer les tensions qui peuvent exister dans le fonctionnement du système et qui en rendent la gestion extrêmement délicate sans qu'en aient encore été évoquées les perturbations récentes.

33 L'ordre dans lequel se pêchent les étangs (l'ordre «théorique " précisent d'un sourire les hommes) est prescrit par les us et coutumes, droit local consigné par écrit et édité à 
différentes reprises. Il suppose, exigerait une concertation, un minimum d'entente ou de courtoisie, on dirait plus volontiers aujourd'hui en Brenne, un « simple bon sens ».

C'est d'aval en amont que les étangs doivent être vidés au fur et à mesure des pêches, le premier étang vidé et pêché en aval recevra les eaux de l'étang situé plus haut et ainsi de suite d'étang en étang. L'eau se voit ainsi chaque fois récupérée, du moins dans le système idéal, de façon à ce qu'il n'y ait que l'eau d'un seul étang qui soit perdue (pour lui ${ }^{7}$ mais pas si l'on considère le système d'ensemble), celle de l'étang le plus en amont qui ne pourra être rempli que par les eaux de pluie et de ruissellement. Ainsi, l'ordre de vidage des divers étangs pour les pêches a-t-il été conçu pour perdre le moins d'eau possible. Car ce dont il s'agit, c'est bien d'en gaspiller le moins possible. Cette notion d'économie de l'eau est essentielle, elle est d'ailleurs dans cette société une constante préoccupation. La gestion des eaux s'opère ainsi à différents niveaux, des petites lignes secondaires d'étangs venant se greffer sur le parcours des grandes lignes, chacune se vidant d'aval en amont jusqu'à l'étang situé sur une ligne importante ou directement dans le ruisseau.

Autre contrainte, liée à ce caractère précieux des ressources en eau que j'ai souligné, et qui prend aujourd'hui, nous allons le voir, une importance considérable avec les perturbations apportées au système d'ensemble du fait même de l'accroissement $d u$ nombre d'étangs, celle du déroulement des pêches dans un temps limité : l'ensemble des étangs doit être vidé et pêché assez tôt dans la saison pour pouvoir bénéficier des pluies d'hiver qui ont lieu jusqu'aux mois de février-mars. Le calendrier, qu'il faut respecter, des dates qui s'échelonnent dans le temps est aussi un élément capital du système. Les pêches des grands étangs débutent en novembre et doivent être terminées à la fin du mois de janvier, l'ensemble des pêches, achevé en mars : tout se passe, ( « tout se joue ", diraient encore les hommes) essentiellement sur quatre mois.

Si les étangs doivent bien se pêcher d'aval en amont, et si le propriétaire de l'étang inférieur doit pêcher le premier son étang, un autre article des usages locaux, l'article 89 , précise toutefois (et il y a là une apparente contradiction qui alimente localement de nombreuses controverses) que celui qui désire pêcher l'étang supérieur devrait avertir un mois à l'avance ${ }^{8}$ de son souhait de vider son étang (et de le pêcher) le propriétaire de l'étang inférieur qui, lui, recevra les eaux; il récupérera l'eau dans le meilleur des cas, ou au moins devra la laisser passer. Mais ce dernier point est déjà précisé d'une phrase, la deuxième, de l'article $88^{9}$; qu'on ait jugé nécessaire à certaines époques d'être plus explicite encore et de laisser une latitude à l'amont est sans aucun doute significatif des éventuelles réticences de l'aval.

Outre les conventions particulières très anciennes qui peuvent exister entre certains étangs que les propriétaires successifs se doivent de respecter, toutes sortes d'arrangements ont lieu, dont je ne peux présenter ici la diversité, mais qui sont nécessaires au fonctionnement même du système ${ }^{10}$. Et en premier lieu, s'il n'est plus vraiment question de respecter ce fameux mois d'avance vu le nombre de nouveaux étangs créés, les propriétaires tentent de s'entendre sur l'ordre des pêches et, pour certains d'entre eux, dont les étangs jalonnent le même ruisseau, de planifier précisément les dates de pêches qui seront reprises d'année en année ou reconsidérées ensemble. Éventuellement pour faciliter les pêches de certains étangs ou pour des convenances personnelles, l'ordre aval/amont pourra être inversé, momentanément ou définitivement. 

l'eau ». Ce principe joue à différents niveaux de la gestion de l'eau. Mais s'il est admis, et unanimement énoncé, il n'est pas acquis pour autant. Et tout un jeu se mène autour de l'eau qui se révèle, dans cette société d'étangs, comme dans la société oasienne, un langage privilégié. C'est par l'eau en effet que l'on s'exprime, parfois règle ses comptes. Le langage de l'eau est le plus efficace parce que forcément entendu. De mauvais rapports de voisinage, ou de simples relations conflictuelles qui n'ont rien à voir avec l'eau, peuvent aussitôt se répercuter dans la gestion de l'eau : il y a là une opportunité de réplique immédiate, bien concrète ou de revanche. Et les hommes pour cela disposent de différents moyens : depuis l'amont, ne pas retenir l'eau alors qu'un étang en aval doit être mis en pêche, temporiser, jouer sur les nerfs de ceux qui sont en aval et qui doivent tenir l'étang prêt, c'est-à-dire suffisamment vide pour la pêche et qui attendent le négociant à l'aube. Depuis l'amont toujours, menacer de ne pas envoyer un courant d'eau au moment de la pêche de l'étang en aval, alors que l'arrivée d'un courant d'eau est essentielle pour le poisson amassé dans très peu d'eau pour la pêche (l'importance de l'opération vient d'être soulignée). Ou encore, envoyer brutalement l'eau d'un étang, alors qu'en aval on est en train de pêcher... Et autant un courant d'eau, un filet d'eau est important pour qu'une pêche se déroule bien, autant une arrivée d'eau soudaine, de ne serait-ce que quelques hectares d'eau, peut perturber une pêche et en compromettre le déroulement. Mais les représailles de l'aval sont possibles : refuser que l'eau passe, faire en sorte que l'étang en amont ne parvienne pas à se vider, et ainsi retarder une pêche, faire attendre, faire pression avec l'eau. privilège ou le pouvoir de l'amont sur l'aval, la dépendance de l'aval par rapport à l'amont, tout comme d'ailleurs dans le système d'irrigation précédemment décrit.

Aussi le système n'est-il viable que par la force des tractations, des négociations qu'il suppose et qui seules rendent son fonctionnement possible et efficace. Il ne saurait être considéré sans les multiples arrangements qu'il exige. Décrire le réseau hydrographique comme un réseau " peu organisé $»^{11}$ du seul fait que l'eau qui s'écoule sur un plateau de faible inclinaison est retenue "naturellement " par cuvettes et coulées $^{12}$ nous ramène à une époque originelle... Depuis l'intervention des hommes, 
sans doute à l'époque monastique, plus fermement au XVe siècle, le réseau hydrographique et le système qui lui est associé présentent une grande complexité non seulement dans les aspects les plus matériels, comme la situation et l'agencement des plans d'eau, mais dans la gestion même, complexité qui se trouve considérablement accrue aujourd'hui. Dom Bascher (1980: 105) parle d'une véritable "politique d'étangs " pour l'époque de Guillaume de Fougères, abbé de Fongombault et prieur de Loups au XVe siècle : elle ne pouvait aller sans l'instauration d'une réglementation.

On comprendra dès lors aisément que le système puisse être profondément perturbé, les tensions exacerbées et devenues intolérables avec le phénomène récent de la création, taxée de façon significative d' " anarchique», par des propriétaires "étrangers à la Brenne »-et l'on veut précisément dire ignorant les usages-, de nouveaux étangs venus se loger tout en amont, ou s'intercaler au sein des anciennes lignes d'étangs où, on l'a vu, on avait déjà bien du mal à réussir la coordination ${ }^{13}$.

Créer un nouvel étang, s'il a moins de trois hectares, n'est pas soumis à autorisation et l'on ne procède pas selon les règles de l'art : il suffit de faire appel à une entreprise qui intervient avec bulldozer et pelleteuse, racle, éventuellement creuse, puis repousse la terre pour ériger des digues qui encadrent géométriquement les plans d'eau. Les nouveaux étangs sont parfois établis n'importe où, "en dépit du bon sens", commentent les hommes, en des lieux ne bénéficiant pas d'eaux d'écoulement, sur des buttes, il n'est pas tenu compte des niveaux, les arrivées d'eau ne sont pas calculées. Les ouvrages eux-mêmes souvent ne sont pas conçus pour les déversements d'eau ${ }^{14}$. Tous les observateurs ${ }^{15}$ ont pu le constater, le phénomène existe depuis déjà trois décennies.

Et, contrairement aux terroirs oasiens, il n'existe pas ici de groupe qui ait une quelconque emprise sur le territoire, emprise qui aurait peut-être permis, pendant un temps au moins, de contrôler ou de ralentir le phénomène. L'aménagement des étangs n'a pas exigé de travaux d'envergure ou l'installation de grands ouvrages qui auraient justifié la pérennité d'une organisation collective forte. Nombre de nouveaux propriétaires n'hésitent pas à arguer qu'on est en terre de propriété privée et à clamer qu'ils entendent bien faire ce qu'ils veulent, comme s'ils annonçaient leur volonté d'ignorer la dépendance, nécessaire pourtant, qu'implique l'inscription de leurs nouveaux étangs dans le réseau hydrographique ancien.

Établis sans analyse préalable du meilleur emplacement topographique, ces étangs le sont sans considération pour l'organisation existante, sans estimation des conséquences pour les anciens étangs, conséquences dont les hommes d'étangs ${ }^{16}$, eux, prennent la juste mesure. Ils évaluent de façon précise ce que représente le détournement des eaux par les étangs installés en amont; leur création capte en partie les eaux de ruissellement du bassin versant qui venaient remplir les étangs anciens. Aux yeux des propriétaires des étangs plus anciens, elle est vécue confusément ou explicitement comme une usurpation.

Et même s'ils parviennent à ce que l'ordre de vidage soit respecté, et s'ils récupèrent ainsi les eaux des nouveaux étangs, certains prennent cependant en compte la déperdition d'eau qu'ils subissent malgré tout: ils en viennent à calculer les pertes d'eau dues à l'évaporation pendant l'été dans les étangs créés en amont; l'eau récupérée après vidage de l'amont sera moins importante que l'eau qu'ils auraient reçue avant la création d'étangs...

La fondation de nombreux étangs rejaillit également sur la gestion d'ensemble du système. L'organisation du vidage des étangs pour les pêches et la coordination des 
pêches se voient perturbées et souvent compromises, que les nouveaux propriétaires méconnaissent un temps les règles, ouvrent les bondes de leurs étangs sans prévenir les propriétaires en aval ou, ce qui est plus grave bien sûr, que, revendiquant être maîtres chez eux, ils décident délibérément de ne pas considérer les usages en vigueur et l'ordre habituel de vidage des étangs et vident quand ils le veulent. On parle alors de vidage "sauvage", terme éloquent qui renvoie en effet à un comportement hors normes. Le fait que de surcroît, mal conçus, ces nouveaux étangs se remplissent parfois très mal, amène leurs propriétaires à vouloir pêcher plus tôt, parfois très tôt dans la saison, afin de parvenir à les remplir ensuite même si leurs étangs sont situés très en amont, sans respecter donc la priorité nécessaire de l'aval et restituer aux autres l'eau qu'ils ont captée. C'est ainsi toute l'efficacité technique du système qui se voit remise en cause.

Mais la méconnaissance par certains propriétaires des usages et des obligations qui leur incombent ne concerne pas seulement l'ordre de vidage des étangs et le respect de cet ordre. Il concerne aussi les ouvrages eux-mêmes, leur entretien et l'indispensable curage des fossés : les négligences à propos de ce dernier point ${ }^{17}$, ont des conséquences importantes, certes au moment des crues, mais aussi pour l'écoulement et le vidage dans des conditions normales.

Autre facteur de perturbation dans le vidage enfin, le développement de la chasse au gibier d'eau qui a amené une très forte valorisation des étangs dont l'accroissement du nombre pourrait d'ailleurs être mis en rapport avec ce phénomène. Outre qu'il suscite l'apparition de conflits d'usage entre chasseurs et agriculteurs-éleveurs, ce développement de la chasse crée une opposition d'intérêts manifeste sur certains groupes d'étangs entre différents propriétaires, selon que les uns privilégient la chasse du gibier d'eau, les autres, la pisciculture; les premiers voulant pêcher le plus tard possible, pour qu'eux-mêmes ou les locataires du plan d'eau puissent en jouir le plus longtemps possible, au moment du passage des oiseaux, les seconds, le plus tôt, pour les raisons que je viens d'évoquer, des raisons évidentes de remplissage. Cela provoque une pression soutenue sur le calendrier des pêches... Pression qui s'est vue exacerbée par des périodes de sécheresse: la crainte est vive, parfois même obsessionnelle pour beaucoup de propriétaires, de ne pas parvenir à remplir suffisamment leurs étangs.

Mais cette tension est, par la force des choses, communicative, justement du fait que les eaux forment système. Qu'un nouveau propriétaire, le plus en amont, et anxieux de ne pas parvenir à remplir ses étangs qui ne peuvent bénéficier d'eaux de ruissellement, décide de pêcher en début de saison avant les autres, les hommes essaieront par tous les moyens en premier lieu de le convaincre de changer d'avis, de s'inscrire dans l'ordre des pêches, puis, s'ils n'y réussissent pas, ils tenteront de stocker cette eau, ou encore d'avancer eux-mêmes leurs dates de pêche. Souvent, ils n'y parviendront pas : l'eau est alors pour eux perdue. Parfois enfin, il peut y avoir une véritable coupure entre l'amont et l'aval. Un certain nombre de propriétaires d'étangs récents, situés tout en amont, se libèrent de toute dépendance par rapport à l'aval. Ils s'arrangent cependant entre eux pour ne pas perdre l'eau de leurs étangs. Cela donne lieu alors à de nouveaux aménagements techniques, en amont comme en aval.

La création par ailleurs de nombre d'étangs insérés au milieu des autres, tout au long d'une ligne, même en aval, amène aussi, à juste titre, le propriétaire situé tout en amont -le plus respectueux des règles soit-il, le plus concerné et préoccupé selon l'usage de faire bénéficier l'aval de son eau- à juger intolérable d'attendre au-delà 
d'une certaine date que tous les étangs soient vidés en aval jusqu'au dernier avant lui. Il ne peut être soumis aux dates de tous les autres, devenus trop nombreux, sans intervenir et il sera amené à faire pression sur tout l'aval pour que l'ensemble des étangs soit pêché suffisamment tôt, avant une date qu'il juge la date limite et que tous d'ailleurs conviendront à juger telle.

Pour conclure, dans les deux exemples que j'ai choisi de présenter, société oasienne et société des étangs, progressivement ou plus brutalement, et du fait, dans les deux cas, à des époques fort différentes, de l'émergence ou du développement de pratiques individualistes qui vont à l'encontre du fonctionnement d'un système basé sur la concertation, les hommes perdent la maîtrise des choses, si tant est qu'il y ait jamais eu de véritable "maîtrise ", dans le cas de la société brennouse. Dans cette dernière société, il n'y a pas en effet, à moins de remonter bien sûr à l'époque monastique, de mainmise d'un groupe structuré sur un espace, comme c'est le cas des groupes lignagers dans la société oasienne; tout au plus, un droit coutumier vient-il suggérer, recommander des règles à observer; mais a-t-il jamais eu pouvoir coercitif ?

Dans les deux exemples, il vient un moment où le fonctionnement du système technique est bouleversé, l'efficacité technique du système, menacée; pendant un temps les hommes tentent d'intervenir, puis ils n'ont pas d'autre choix que de s'adapter. Alors que dans la société oasienne où les transformations ont eu lieu bien plus tôt, et où les hommes, de guerre lasse ont dû démissionner, le système technique, on l'a vu, continue sa propre vie au sein du social mais de façon autonome et se trouve un moment comme coupé de la société réelle, dans la société brennouse où il nous est donné d'observer le processus de changement en cours, on peut voir au début les hommes, toujours individuellement, intervenir, essayer de réduire les aléas, de parer aux vidages "sauvages", ou de se plier à ces nouvelles contraintes, pour sauver la marche du système.

Suivant les lignes d'étangs, suivant les propriétaires concernés, les situations peuvent être très différentes. Sur certaines lignes, mais ce n'est là qu'un exemple, on peut voir comment s'exerce subtilement l'influence de grands propriétaires, issus de familles puissantes localement, sur les petits agriculteurs devenus propriétaires d'étangs. Ces derniers peuvent aussi bien connaître les usages sans pour autant les respecter s'ils estiment que cela ne leur convient pas, et il y a là parfois confusément un refus de se soumettre au pouvoir des grands propriétaires, ou tout simplement de devoir rendre des comptes à l'aval. Ailleurs, les hommes vont trouver les propriétaires de l'amont ${ }^{18}$, tentent de dialoguer, d'expliquer, d'informer les nouveaux venus du fonctionnement du système, d'en rappeler les principes. De nouvelles tractations se déroulent, des compromis se font, les hommes peuvent être amenés à resserrer dans le temps le calendrier des pêches de façon à satisfaire les exploitants de l'amont pour les dates, et ainsi récupérer leur eau. Tout est fait pour ne pas risquer de perdre l'eau des étangs nouvellement créés dont les propriétaires refusent de considérer l'insertion dans l'économie globale du système. La pression reste cependant très forte, la complexité du système s'en trouve accrue. Les hommes en ont conscience et la façon dont ils parlent du vidage des étangs et du fonctionnement concret du système est à cet égard éloquente.

Mais contrairement à la société saharienne où les hommes avaient à gérer, entre eux, le système technique d'irrigation (hormis, pour certains des villages, durant la période 
d'ingérence de l'administration coloniale ${ }^{19}$ ), on est avec la Brenne dans une société où interviennent différents acteurs avec, chacun, des enjeux qu'il n'y a pas lieu d'analyser ici. La gestion des étangs n'est pas considérée comme la seule affaire des hommes d'étangs.

Avant les années 1980 déjà, des voix s'étaient fait entendre, notamment dans les communes les plus touchées par la création de nouveaux étangs. Les élus locaux s'insurgeaient contre ce qu'ils nommaient significativement «la prolifération anarchique » des nouveaux étangs. Des débats avaient lieu, réunissant élus locaux, représentants de la Chambre d'Agriculture et aussi bien des syndicats d'agriculteurs et des exploitants piscicoles.

Les Rencontres sur l'environnement en Brenne en 1987 font état d'une inquiétude partagée face à ce «mitage» de l'espace. Le terme d'" anarchique» revient fréquemment. On estime nécessaire désormais la mise en place de dispositifs réglementaires et législatifs.

Si la loi sur l'eau de 1992, qui était devenue indispensable du fait de la multiplicité des textes de référence, avait été diversement appréciée et jugée insuffisante quant aux procédures d'autorisation de création de plans d'eau, elle avait provoqué de vives réactions à ses énoncés sur les procédures d'autorisation de vidage. Les exploitants d'étangs voyaient là une atteinte au système traditionnel ("séculaire " insistaient-ils) de pêche. Des tractations avaient lieu entre la Mission Inter-Services de l'Eau (MISE) et le Syndicat des exploitants d'étangs ${ }^{20}$ qui affirmait très fermement ses positions. Un accord semblait s'être fait un moment sur de simples déclarations de valeur trentenaire à remettre à la MISE qui devaient préciser « les périodes » de vidage prévues, mais le syndicat estimait impossible, du fait des aléas, la déclaration d'une date sûre et qui aurait dû être identique d'année en année. Un décret d'août 1999 venait calmer les débats : l'antériorité de l'activité piscicole reconnue sur un étang permet désormais une autorisation définitive de vidage.

61 Dans son projet de charte révisée, à l'article 9, le Parc Naturel Régional énonçait en juillet 1997, vouloir « agir pour une gestion concertée de l'eau et des étangs ». Se posant en médiateur entre l'ensemble des partenaires ("propriétaires, pisciculteurs, chasseurs, naturalistes, scientifiques, touristes »...), il annonçait son souhait d'élaborer un nouveau " code de bonne conduite ", vocabulaire qui avait fait grincer les dents ou sourire $^{21}$.

Le Syndicat Intercommunal pour l'Assainissement et la Mise en Valeur de la Brenne (SIAMVB) enfin, en la personne de son président, également maire de la commune la plus importante de Brenne prenait les choses en main. Sous forme d'un dépliant illustré, un code de «bonnes pratiques » cette fois, "pour une gestion concertée des étangs de la Brenne » était édité au printemps 2000 et envoyé à tous les propriétaires d'étangs. Il rappelle les grands principes du coutumier et notamment celui de l'ordre du vidage de l'aval vers l'amont, et le respect des dates limites pour la saison des pêches afin de "permettre le remplissage pendant la saison de plus grande pluviosité ». "La concertation entre exploitants est donc nécessaire, est-il souligné, afin de s'entendre sur les dates de vidange. » Mais le SIAMVB ne compte pas s'arrêter là. S'appuyant sur une solide documentation statistique et cartographique, il lance avec le soutien du Syndicat des Exploitants Piscicoles une enquête générale sur les étangs. L'objectif du Président est clair : intervenir largement et fermement dans la gestion hydraulique, à la fois pour contrôler et limiter la création d'étangs et pour faire respecter les usages ${ }^{22}$. 
ten posant la question du véritable statut d'un droit coutumier dans la société moderne, on avait envisagé un moment d'actualiser certains de ses articles, entreprise jugée impossible par la Chambre d'Agriculture qui eut été sans doute contrainte alors de réviser l'ensemble du recueil et non pas seulement les articles concernant les étangs. Pensant que cela pouvait être utile, certains y réfléchissaient, comme cet ancien notaire, tout autant homme de terrain, grand propriétaire d'étangs, qui fut longtemps président du Syndicat des exploitants d'étangs de Brenne et qui a consacré une grande part de sa vie à l'exploitation piscicole ${ }^{23}$. L'article 89 dans le contexte actuel lui parait inadapté ${ }^{24}$.

« [...]. Il est facile de comprendre, note-t-il, que si chaque exploitant mettait son étang en vidange à la date qui lui convient, sans se préoccuper des exploitants des étangs situés en aval, ce serait la plus grande pagaille et cela, même si les exploitants des étangs du dessous étaient prévenus longtemps à l'avance. Pour une bonne gestion des étangs de Brenne, il est donc essentiel que de bonnes pratiques soient rigoureusement observées, il faut redonner toute sa force au seul article 88 $\mathrm{du}$ recueil des usages locaux. Les exceptions à ce principe sont bien entendu possibles, mais elles ne peuvent résulter que d'un commun accord entre les exploitants d'étangs concernés. Pour y parvenir il faut que soit organisé à l'initiative de celui qui désire un arrangement différent du principe, une concertation entre les exploitants des étangs qui se vident les uns dans les autres. À défaut d'accord, l'article 88 des usages locaux devra être respecté. »

Le respect de la règle ou les arrangements toujours possibles : tout l'esprit du système est là en effet, dans sa souplesse même. Et là réside la difficulté. Car l'on ne saurait rigidifier la concertation.

L'ensemble des propriétaires d'étangs auront un jour ou l'autre à régler ce paradoxe : être en terre de propriété privée, être maîtres chez eux, et, du fait même de l'appartenance de leurs étangs à un système large, admettre la dépendance et la solidarité nécessaire qu'il impose. À eux ensuite de trouver au jour le jour les ajustements qui concourent aussi à l'efficacité du système.

Il faut que les perturbations que connaît le système soient graves pour que tout le monde s'en alarme, et tente d'intervenir; l'observation de la région en cette période de crise à laquelle les hommes sont confrontés est particulièrement instructive. Autant qu'ils le peuvent, ils essaient de préserver l'efficacité du système, de réduire ce décalage flagrant entre système technique et société plus large. D'autres préoccupations, écologiques notamment, se sont fait jour et sont désormais prises en compte.

67 Mais une situation de crise ne peut s'installer durablement, on l'a vu avec la société saharienne, on le voit bien avec cet exemple aujourd'hui de la société brennouse : par paliers, avec des tâtonnements, des soubresauts, tous les efforts, individuels ou organisés, vont vers un réajustement $d u$ système technique, un réajustement à l'ensemble d'une société locale qui s'est déjà transformée, elle-même aura à faire d'inévitables concessions. Nous sommes là dans la dialectique des rapports entre le technique et le social -entre différents ordres du social. 


\section{BIBLIOGRAPHIE}

Assier-Andrieu, Louis (ed.)

1990. Une France coutumière. Enquête sur les « usages locaux » et leur codification (XIXe-XXe siècles).

Paris : Éditions du CNRS.

Barrère, Elisabeth

1983. « Approches de la pisciculture en Brenne (Indre) », Rapport dans le cadre d'un protocole MNHN. Ministère de l'Environnement.

Bédoucha, Geneviève

1987. «L'eau, l'amie du puissant ». Une communauté oasienne de l'extrême Sud tunisien. Paris : Éditions des Archives contemporaines.

1993. "The Watch and the Waterclock. Technological choices/social choices ", pp. 77-107, in

P. Lemonnier (ed.), Technological choices. Transformation in material cultures since the Neolithic.

Londres/New York : Routledge.

2000. "Autour des étangs, la société brennouse ", Aesturia $1:$ 89-112 (numéro spécial, Marais et zones humides, Cultures, sociétés et territoires).

Bérard, Laurence

1982. Terres et eaux en Dombes. Technologie et droit coutumier. Lyon/Paris : Presses Universitaires de Lyon/Éditions de la Maison des Sciences de L'Homme.

Bidault, Magali

1998. Le bassin versant de Picadon : un exemple des dynamiques hydrauliques et paysagères en Brenne Centrale. Mémoire de maîtrise. Tours : Université François Rabelais, Département de Géographie.

Chambre d'agriculture de l'Indre

1967. Usages locaux à caractère agricole du Département de l'Indre codifiés par la Chambre d'Agriculture. Châteauroux : Imprimerie Laboureur et Cie.

Cresswell, Robert

1996. Prométhée ou Pandore? Propos de technologie culturelle. Paris : Éditions Kimé.

Dom Bascher, Jacques de

1980. Le Prieuré Sainte-Madeleine de Loups, première fondation de l'Abbaye de Fontgombault 1096-1791.

Châteauroux : Imprimerie Laboureur.

Guillemot, Hélène

1988. « La Chaîne des Grandes Fourdines ». Essai de description technique, juridique et économique d'une chaîne d'étangs en Brenne (Indre). Mémoire de DEA d'Anthropologie Sociale. Paris : EHESS.

Jubault, Patrick

1991. La prolifération des étangs. Mémoire de l'École Nationale Supérieure Agronomique de Rennes. Parc naturel régional de la Brenne.

Tardivon, Jacqueline

1991 Usages et coutumes en Brenne. s.l. : Royer (« Mémoire vive »).

- Loi sur l'eau n92 - 3 du 3 janvier 1992. 
- Décrets n 93-742 relatif aux procédures d'autorisation et de déclaration selon l'article 10 de la loi sur l'eau n 92-3 du 3 janvier 1992, et 93-743 du 29 mars 1993 relatif à la nomenclature des opérations soumises à autorisation ou à déclaration en application de l'article 10 de la loi.

- Décret n 99-736 du 27 août 1999 modifiant le décret nº 93-743 du 29 mars 1993.

\section{NOTES}

1. Il va de soi que la distinction peut avoir une fonction méthodologique à certaines étapes de la recherche mais n'oppose nullement des essences : le technique est bien partie intégrante du social.

2. R. Cresswell me l'accorde volontiers (communication personnelle).

3. Pour une analyse de ces conséquences, je renverrai à Bédoucha (1993).

4. Si l'on considère l'efficacité du système pris dans son ensemble, il y a bien perte d'eau pour le propriétaire auquel elle est destinée, mais par infiltration, tout au long de son parcours, l'eau profite aussi largement aux terres riveraines.

5. Pour les arguments en faveur de cette assertion, je renverrai à Bédoucha (2000).

6. La région est essentiellement productrice et depuis longtemps de carpes, mais aussi de brochets et perches, tanches et gardons. Une grande partie de la production de carpes estimée à 1200 tonnes est exportée vers l'Allemagne.

7. Cet inconvénient majeur est largement compensé par l'avantage, unique pour l'étang le plus en amont, de ne pas être peuplé de brochets prédateurs que le propriétaire peut juger indésirables, ou de redoutés poissons-chats. Ce sont les étangs le plus en amont qu'il était d'usage de réserver à la ponte et aujourd'hui au grossissement des alevins. 8. L'usage voulait aussi que cela se fît par lettre recommandée avec accusé de réception. Les propriétaires de certains étangs continuent d'y avoir recours même si dans le contexte actuel, les réactions de l'aval sont parfois vives.

9. « Si (le propriétaire de l'étang inférieur) ne veut pas le pêcher, il doit au moins baisser le niveau de manière à rendre possible la pêche de l'étang supérieur ».

10. Il en est de même dans une autre région d'étangs, au demeurant fort différente, la Dombes. L. Bérard (1982) analyse de façon détaillée tous les arrangements possibles au sein d'un groupe spécifique d'étangs.

11. Comme l'ont fait certains travaux, par ailleurs très instructifs (Barrère 1982, Guillemot 1988).

12. Outre le fait que la construction des ouvrages adaptés ne se réduit pas à barrer les coulées de terrain à travers lesquelles l'eau s'écoule par déclivité naturelle.

13. Nous avons des informations précises sur la création d'étangs dans un bassin versant particulier grâce au mémoire de M. Bidault (1998). Il y aurait eu, sur ce bassin versant, plus de créations d'étangs entre 1975 et 1988 (13\% d'augmentation) que du Moyen Âge à 1975; et plus entre 1988 et 1997 (51 \% d'augmentation) qu'entre 1975 et 1988...

14. On est loin de cette époque, au XVe siècle déjà, où, les étangs se multipliant, on avait dû avoir affaire à des spécialistes, les bessons, pour juger de l'emplacement le plus approprié à la création d'un étang. J. Tardivon (1991) le souligne très justement, s'appuyant en cela sur les travaux de Dom Bascher (1980).

15. Et notamment Jubault (1991) qui en présente une étude très complète. 
16. J'entends par « hommes d'étangs », outre les propriétaires exploitants piscicoles, tous ceux qui ont affaire à la gestion concrète de l'eau, gardes et ouvriers agricoles mais aussi éventuellement agriculteurs chargés de suivre de près l'exploitation d'étangs. 17. Les articles 62 à 64 des usages locaux sont clairs : si le ruisseau d'écoulement des eaux à travers les fonds inférieurs est présumé être la propriété des riverains, ceux-ci ne doivent pas en entraver le libre écoulement et surtout ils doivent laisser le propriétaire de l'étang en effectuer le curage, et supporter que les curures soient déposées sur les bords. Aucun article toutefois ne vient recommander un curage régulier, souvent négligé d'ailleurs, par le propriétaire de l'étang qui n'est pas toujours non plus dans les meilleurs termes avec le propriétaire des terres du fonds inférieur... 18. Tout comme dans le système d'irrigation précédemment décrit, lorsque les hommes, voyant l'eau qui arrive dans leur jardin s'affaiblir ou cesser de couler, ils remontent vers l'amont pour trouver le responsable du détournement d'eau.

19. On ne peut négliger l'influence, après l'Indépendance, des techniciens modernistes dépêchés sur place.

20. Ancienne dénomination du Syndicat des exploitants piscicoles.

21. «Il élaborera [...] un nouveau 'code de bonne conduite' qui définira les règles à préconiser pour un bon usage de l'eau et de la gestion de l'espace en Brenne. Seront notamment visés les problèmes posés par la création de nouveaux étangs (règles techniques à respecter, système hydraulique, vidange, interdépendance des étangs, intégration esthétique et paysagère, etc. [...] Les préconisations du 'code de bonne conduite' pourraient également fournir un cadre de référence à l'administration pour faciliter l'application de certaines réglementations comme celles issues de la loi sur l'eau du 3 janvier 1992 pour la création des étangs. »

22. Tout cela mériterait plus ample développement mais ne saurait être détaillé dans le cadre de cet article. Je préciserai par ailleurs pour une société en mouvement comme la société brennouse, que les informations réunies dans cet article datent, pour les dernières, du printemps de l'année 2000.

23. Je remercie Maître Moreau de m'avoir communiqué ses réflexions qu'il avait mises par écrit.

24. Loi écrite locale, «l'usage ne cesse pas pour autant sa vie pratique obéissant à l'évolution concrète des relations sociales " : le propos d'Assier-Andrieu (1990:10) se voit parfaitement illustré par cet exemple.

\section{RÉSUMÉS}

Après avoir démontré en quoi une analyse comparative entre deux sociétés aussi contrastées qu'une société à étangs en zone humide et une société pratiquant l'irrigation en zone aride est légitime, l'auteur examine les discordances, en termes d'efficacité, apparues ici et là entre système technique et société, les perceptions et les façons différentes, au sein de chacune de ces sociétés, de réagir face à ces discordances parfois flagrantes et difficilement supportables pour les hommes, les ajustements qui à un moment ou à un autre nécessairement s'opèrent. 
Momentary dissonances : a comparative analysis.

The author asserts the legitimacy of comparing two strongly contrasted societies : the one living off of ponds in an humid zone, the other practising irrigation in an arid zone. She first examines the differences in efficiency appearing here and there between system of technics and society, then the perceptions and different reactions in each of them faced with the sometimes flagrant discordances, difficult for men to bear and, finally the adjustments which eventually will occur.

\section{Discordancias momentáneas. Análisis comparativo.}

¿ Resulta legítimo llevar a cabo un análisis comparativo entre dos sociedades tan contrastadas que la primera cuenta con estanques en zona húmeda, en tanto que la segunda sociedad practica la irrigación en zona árida ? Después de dar una respuesta afirmativa y bien argumentada a esta cuestión, el autor pasa a examinar las diferencias que en términos de eficacia aparecen aquí y allá entre sistema técnico y sociedad; las percepciones y las formas diferentes que se registran, en el seno de cada una de estas dos sociedades, de reaccionar frente a discordancias a menudo flagrantes, y que las personas difícilmente pueden soportar; y los ajustes que en un momento $u$ otro acaban por producirse.

\section{INDEX}

Keywords : Brenne, oasis, Sahara, damp zone, irriguation, pond, technical system, unwritten law, water control

Mots-clés : droit coutumier, étangs, gestion des eaux, irrigation, système technique, zone humide

\section{AUTEUR \\ GENEVIÈVE BÉDOUCHA}

UMR 8098, « Technique et culture », CNRS, 27 rue Paul Bert, 94204 Ivry-sur-Seine Cedex 